\title{
Pérdida de valores morales en alumnos de la zona rural
}

\section{Loss of moral values in students from rural areas.}

\author{
Gómez-Medina, María de los Ángeles; Rivas-García, Julio Antonio; \\ Martínez-Andrade, Erick; Villalobos-Maradiaga, Edixia María; \\ Zuniga-Gonzalez, Carlos Alberto; Editor Academico Dr. Angel Sol \\ Sanchez
}

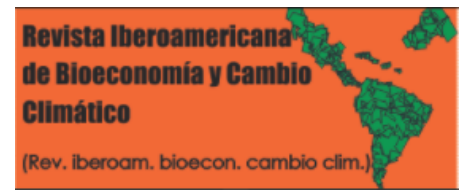

\author{
María de los Ángeles Gómez-Medina \\ mariade.gomez@post.unanleon.edu.ni \\ MINED-Villanueva, Nicaragua \\ Julio Antonio Rivas-García \\ UNAN-León, Nicaragua \\ Erick Martínez-Andrade \\ UNAN-León, Nicaragua \\ Edixia María Villalobos-Maradiaga \\ UNAN-León, Nicaragua \\ Carlos Alberto Zuniga-Gonzalez \\ Universidad Nacional Autónoma de Nicaragua, León., \\ Nicaragua \\ Editor Academico Dr. Angel Sol Sanchez \\ Colegio de Postgrados Mexico, Mexico
}

Revista Iberoamericana de Bioeconomía y Cambio Climático

Universidad Nacional Autónoma de Nicaragua, León, Nicaragua ISSN-e: 2410-7980

Periodicidad: Semestral

vol. 2, núm. 1, 2016

czuniga@ct.unanleon.edu.ni

Recepción: 14 Febrero 2015

Aprobación: 04 Mayo 2016

URL: http://portal.amelica.org/ameli/journal/394/3941750029/

DOI: https://doi.org/10.5377/ribcc.v2i1.5709

Autor de correspondencia: mariade.gomez@post.unanleon.edu.ni
Resumen: El estudio se centró en un intento para discutir el tema sobre la perdidas de valores en la zona rural, en este campo de la educación que mucha veces se toma en cuenta la parte emocional e intelectual, en que refiere al campo en la familia y en la escuela, no tiene como objeto reprimir las emociones, sino conocerlas, saberlas utilizar para el desarrollo y la motivación personal y también aprender a controla. Es menester estar pendiente de los valores humanos que poseemos dando un paso gigantesco haber introducido seriamente en el campo de la personalidad y de la educación en un mundo globalizado y compuesto por emociones y aptitudes pocos humanitaria, que antes se reservaba más bien a la literatura y al arte. Así que la formación de los valores morales se impone en nuestros días por la compleja situación que vivimos a nivel internacional, de ahí la necesidad de priorizarla en las instituciones educacionales de nuestro país, pues es la niñez, adolescencia y juventud son los sector priorizado en la sociedad para la transformación que se necesita en el profesional, cuya actuación debe estar en consonancia con la máxima aspiración de formar hombres responsables y comprometidos con los demás y con una concepción humanista.

Palabras clave: Enseñanza, Valores morales, Escuela, Estudiantes, Nivel de estudio.

Abstract: The study focused on an attempt to discuss the issue of the loss of values in rural areas, in this field of education that often takes into account the emotional and intellectual part, in that it refers to the field in the family and in The school does not aim to repress emotions, but to know them, to know how to use them for personal development and motivation and also to learn to control them. It is necessary to be aware of the human values that we possess, taking a gigantic step, having seriously introduced the field of personality and education in a globalized world made up of emotions and aptitudes that are not very humanitarian, which before was reserved more for literature and to art. So the formation of moral values is imposed in our days due to the complex situation that we live at the international level, hence the need to prioritize it in the educational institutions of our country, since childhood, adolescence and youth are the prioritized sector in society for the transformation that is needed in the professional, whose performance must be in line with the highest aspiration to train 
responsible men and committed to others and with a humanistic conception.

Keywords: Teaching, Moral values, School, Students, Study level.

\section{INTRODUCCIÓN}

En la formación del seres humanos que vive en zonas rurales donde los profesores, padres de familia y autoridades locales deben darle un papel muy importante a la formación de acciones y orientaciones valorativas en sus clases, vida social y legal. Todas ellas estarán vinculadas al valor del conocimiento que es objeto de aprendizaje, cualidad y conducción que le confiere su valor social.

La educación de valores muchos exige del proceso docente - educativo el formar a los niños con una concepción científica del mundo y con un sistema de valores que le dé sentido a sus vidas; exige, por tanto, enseñar a vivir a las nuevas generaciones a partir de los ideales que la sociedad de hoy se propone. Hay que tener en cuenta que los niños nacen con cualidades morales determinadas; no nacen veraces, ni embusteros, ni afables, ni malignos, ni vagos, ni trabajadores. Esto lo adquieren en el medio en que se desarrollan

Por lo que la familia, como institución, expresa el conjunto de valores de la sociedad en la que tiene lugar, así como cumplimenta toda serie de normas y patrones que se reproducen de generación en generación. Desde luego, que cada una de estas últimas enriquece a la que le precede al fortalecer rasgos de esta y modificar otros.

Entre los valores que se rigen en la nueva sociedad es preciso destacar el humanismo, la honradez, el colectivismo, el patriotismo, el antiimperialismo, el internacionalismo, la solidaridad, el espíritu de sacrificio, justicia y modestia, así como una nueva actitud ante el trabajo y la posición de la mujer en la sociedad. Por lo que cada unidad integradora de lo afectivo y racional donde se expresa la gran importancia de lo afectivo en la educación moral. Todos los elementos que se integran en la personalidad tienen una naturaleza racional y efectiva.

Dicha unidad de la actividad con la comunicación que crea un clima adecuado de compresión mutua entre los individuos. Por lo instructivo con lo educativo, junto con el proceso de interiorización y asimilación de conocimientos y habilidades, debe ir produciendo cambios en la conducta de la personalidad. Tomando en cuenta la elevación del carácter científico del contenido para lograr verdaderos profesionales acorde con los adelantos de la ciencia y la técnica propicios no afecte el comportamiento del ser racional que vive en el campo o zona rural.

\section{REVISIÓN DE LA LiTERATURA}

\section{¿QUÉ ES TENER VALOR?}

Es estimar un objeto, un principio; es ubicar y decidir lo que es más importante en un determinado momento y luchar por eso; es ubicar y jerarquizar los bienes materiales y espirituales en orden de importancia. Pero los conocimientos, aptitudes y valores se adquieren y se forman como resultado de la realidad en que cada sujeto vive y el desarrollo histórico social alcanzado en una época determinada. Esto le corresponde a la escuela desempeñar un papel determinante en la formación de valores, por lo tanto es necesario efectuar

\section{NoTAS DE AUTOR}


transformaciones en el trabajo educativo, por lo que resulta necesario fundamentar que este se oriente de forma tal que permita potencial en cada estudiantes aquellos aspectos que le facilitan conocer de una manera más significativa el momento histórico que viven.

"Nadie pone en duda la importancia del tiempo para la vida de las personas. Nos guste o no, somos seres temporales", según (Zubiri, 2002) esta afirmación expresa una realidad de la vida que todo ser humano debe tomar en cuenta por lo que la conducta es parte importante de poder conducirse bien ante la sociedad fomentado todas las cualidades positiva que nos permites ser parte de ella. Pero en la educación de nuestros jóvenes en especial en la zona rurales ha venido cambiando a través del tiempo en la cual nuestra niñez y juventud vive una vida llena de fantasía y mentiras que provoca las pérdidas de valores

Conjuntamente, la pérdida de valores es uno de los principales desencadenantes de conductas negativas en niñas, niños y jóvenes. Este fenómeno se atribuye en parte, a que valores sociales primordiales no se enseñan ni promueven en la familia, la escuela ni en el medio donde se desenvuelven estas personas. Por lo que este tipo de aptitudes no se ve reflejado en la escuela ni mucho menos en la familia ya que ambos apoya y promover el desarrollo de valores sociales positivos; además, de que su presencia en el sistema educativo nacional contribuye al desarrollo del carácter, la personalidad, y la forma de pensar y actuar la persona. Sino que permiten la enseñanza de los buenos valores; fomentando valores esenciales en la conducción de la vida como la tolerancia y la identidad, entre otros. Para que fortalezcan la voluntad para alcanzar una mejor salud física, equilibrio psicosocial y, por consiguiente, una adecuada calidad de vida.

Además, como expresa (Gimeno, 2008):” La construcción de la construcción de su identidad individual y colectiva, su educación en definitiva, mejoraría si se conociesen y se coordinasen de forma más óptima los tiempos sociales. En este sentido, si el tiempo es vital para las personas, de forma análoga resulta vital su conocimiento, distribución y uso, si de procesos de construcción de la persona, procesos educativos, estamos hablando."

Esta aserción procesa la identidad individual y colectiva, ya que la educación en especial en el campo se está perdiendo por causa de que de las malas interpretaciones de las leyes y el cambio constante de la sociedad a un ritmo muy acelerado, un poco más abierta con menos rigurosidad en especial en el término de la educación. Pero si se conoce y se coordina en una forma más óptima los tiempos, las leyes y las relaciones sociales. En este sentido de forma análoga resulta vital su conocimiento, distribución y uso, tomando en cuenta los procesos de construcción de la persona, siendo el proceso más eficaz es el de educación, donde la idiosincrasia de los pueblos rurales no se vería afectada.

También, José Martí (1963), expresó: “ la educación es la habilitación de los hombres para obtener con desahogo y honradez los medios de vida indispensables en el tiempo que existen, sin rebajar por eso las aspiraciones delicadas y espirituales de la mejor parte del ser humano"

Estos medios que menciona nuestro ilustre poeta están formados por los siguientes elementos humanos:

- Principios o creencias que son los elementos espirituales que todo ser humano está

siempre conectado a un ser supremo ya que la necesidad ella es esencial parte de su ser.

- Relativos o estados de existencia o a modelos de comportamiento esto nos con

lleva a ser parte de la sociedad en que está formado, es decir, no podemos estar fuera de ella ni lo podemos omitir siendo individuo solitario y auto suficiente.

- Trascienden objetos, grupos de personas y situaciones específicas nos lleva a

identificarlo a un grupo específico para poder ser parte de ella, es decir; cada individuo se siente comunicado y bien ubicado y que su pensamiento y aptitudes son similares y por ende se siente cómodo y parte de ella.

- Orientan la elección o evaluación de comportamientos y acontecimientos por lo

que puede ser parte del adiestramiento de otros individuos, formándolos, educándolos y guiándolos a las necesidades que la comunidad necesita.

- Expresan intereses individuales o colectivos siendo parte de la solución de

problemas comunes y a la vez constituir parte de grupos de ayuda que la comunidad necesita. 
¿Los valores se han perdido? Muchas personas (en nuestra opinión erróneamente) plantean que están en crisis y sobre todo en la juventud, la situación se debe analizar pues hay síntomas en alguna parte de nuestra sociedad:

- Perplejidad sobre cuál es el verdadero sistema de valores

- Inseguridad acerca de qué considerar valor y antivalor

- Sentimientos de pérdida de valor de aquello que se consideraba valioso

- Atribución de valor a lo que antes se consideraba antivalor

- Cambio de lugar de los valores en el sistema jerárquico subjetivo.

Ahora bien la formación de valores en las nuevas generaciones depende en grado sumo de la continuidad histórica del proceso revolucionario. Este es un complejo problema en el que concluyen las acciones educativas de diversas instituciones y organizaciones sociales, los medios de difusión masiva, y por supuesto la familia y la escuela.

Igualmente, como lo expresa Rodríguez (2010): “Los valores educativos en su tiempo libre y diferencias significativas a tener en cuenta en función del nivel educativo. El tiempo libre es un tiempo crucial en la vida de los adolescentes y presenta procesos análogos a los experimentados en el tiempo escolar."

Esto da como relevancia que muchas veces los valores se pierden en las zonas rurales por el tiempo libre que los hijos tiene, ya que en los tiempos actuales los jóvenes adquieren otro tipo de distracción como conseguir y aprender conducir motocicletas, fundación de nuevos bares, encuentros con personas alcohólicas, entre otras.

Al mismo tiempo, Martín (2002), señala que:" las actividades que el hombre realiza en este medio es decir, se deben comprender las relaciones que se establecen entre el hombre y su medio ambiente. Y a partir de este conocimiento elaborar juicios y valores personales que lleven al desarrollo de una ética."

No debemos olvidar que muchas veces la niñez, adolescentes y jóvenes que vive en las zonas rurales debe apreciar, estimar y valorar el medio en que se desenvuelve en especial tener contacto con la naturaleza que cuando se mayores ellos tendrá una nueva perspectiva del recurso natural con que cuenta y no perder tiempo en lugares no productivo para su crecimiento integral y personal.

A más de lo antedicho por Lourenço (2005): "La Educación del Campo es el medio por lo cual los grupos pertenecientes al universo del campo buscan su plenitud formativa. Es a través de la Educación para el Campo que el ser humano tiene condiciones de constituirse como un ser social responsable por sus actitudes, por su manera de pensar, de estar en el mundo y de dialogar."

Nos enseñanza que aunque la persona haiga nacido, crecido y vivido en el campo no implica que sus valores humanos se deben de pasar de un extremo a otro, por lo tanto los hijos deben seguir ese modelo de tal manera que sus raíces, enseñanzas y peculiaridad no se debe cambiar o sustituir por modelos que no son apropiados. Por lo general este fenómeno se ve mayormente cuando alguien viaja fuera de su lugar, es decir viaja o permanece en un periodo de tiempo en la ciudad y cuando regresa a un lugar de origen llega con otro estilo de vida. Por lo que otros desean imitar de una manera poco auténtica y con muchas deficiencias en lo social.

Aparte de que Fouratt (2011), expresa que: "muchos migrantes nicaragüenses, invierten en tierras y recursos materiales (enviados como remesas) como parte de una estrategia transnacional de supervivencia familiar. Sin embargo, estas inversiones forman parte de discursos tanto de abandono como de cuido y vínculo familiar. En este artículo se enfatizan las tensiones entre estos discursos para sugerir que, en lugar de causar desintegración o abandono a la familia, las formas de "cuido" a la distancia de los migrantes representan intentos por mantener relaciones familiares cercanas mediante lazos afectivos y materiales con su hogar."

Aunque parece un cuento de hadas esto es una realidad que por el bienestar de la familia muchos emigran a otros países perdiendo un valor muy social como es la familia. Otros por los problemas de emigración tienden permanecer ocultos en países extranjeros, por lo que los progenitores e hijos se sienten muy angustiados y como consecuencia muchos hijos pierden el respeto y amor a sus patriarcas. Por ende, sus valores humanos no son bien reforzados ni corregidos cuando más lo necesitan. 
Aparte de que Fouratt (2011), expresa que: "muchos migrantes nicaragüenses, invierten en tierras y recursos materiales (enviados como remesas) como parte de una estrategia transnacional de supervivencia familiar. Sin embargo, estas inversiones forman parte de discursos tanto de abandono como de cuido y vínculo familiar. En este artículo se enfatizan las tensiones entre estos discursos para sugerir que, en lugar de causar desintegración o abandono a la familia, las formas de "cuido" a la distancia de los migrantes representan intentos por mantener relaciones familiares cercanas mediante lazos afectivos y materiales con su hogar. “

Por lo que los hijos menores son muy vulnerables. Porque el éxito de su niñez depende de cómo los valores son infundados destacando algunos elementos negativos que son muy presentes en su infancia.

1. Los miedos

2. Las mentiras

3. Los celos

4. Los permisos

5. Autoridad y disciplina

Según Segura, M., Mesa, J., \& ARCAS, M. (1997), establecen que:” en la familia y en la escuela, no tiene como objeto reprimir las emociones, sino conocerlas, saberlas utilizar para el desarrollo y la motivación personal y también aprender a controlarlas cuando amenacen desbordarnos."

Esto nos permite ser más consiente en el rol del padre de familia en los valores de su hijos por lo que aunque este lejos son ellos los primeros que deben estar atentos, no solo enviarles dinero sino también inculcarles valores que están practicando fuera de su comunidad y enseñándoles que son los mismo valores que se practican en su zona o lugar en donde vive. Podemos agregar que la comunicación es otro valor importante que debe tener la prole.

Como Conde Gutiérrez Del Álamo, F., \& Santoro Domingo, P. (2012), enuncian:” existe una crisis de valores, deterioro o pérdida de los mismos y para enfrentar tal situación contamos, en primera instancia, con un sistema educacional que responde a las exigencias en la formación de ciudadanos de bien."

Rodríguez, Z. (1985), plantea: "Para la axiología burguesa contemporánea el valor constituye un aspecto particular de la realidad, incompatible con las ciencias naturales y que por ende, no es susceptible de un análisis científico racional”.

Todo lo expuesto por el vemos la crisis de estos valores humanos se va acrecentado debido a la falta de orientación educacional ya que algunos docentes no tiene la capacidad humana de ver estos valores como un punto de lanza para alcanzar el éxito personal que todo individuo tiene derecho; en la búsqueda de la felicidad.

\section{CONCLUSION}

La aspiración de nuestra sociedad es que se formen hombres profesionalmente competentes integrales, éticamente honestos y responsables, con una sólida formación científica y humanística, crítica y comprometida con su entorno, creativa, sensible ante las preocupaciones de los demás y comprometida con la Patria y con la comunidad rural siendo su primera objetivo el lugar de origen. Todo esto permite tener individuos que con una integridad intachable, trabajadora y honesta viviendo en estas comunidades lejanos a la gran ciudad, cuidando, conservando, protegiendo y trabajando la tierra como el primer bien humano que toda sociedad tiene.

En aras de aportar a nuestra Comunidad Educativa es importante darse a la tarea de posibilitar una enseñanza de valores humanos que ellos tienen eliminando todas influencia que otros tenga por viajar o trabajar en lugares donde los valores humanos no son importante. Porque la relación escuela-familia y padres e hijos son la base fundamental para la integración de estos temas que son olvidados, puesto en la balanza como el último lugar en zonas rurales. Por supuesto que proponer es compartir la inquietud de hacer significativos los procesos; no solo proponer es lo natural sino que en actuar. Comprometerse es atreverse a sentirse parte de una acción, es decir que nuestro aporte es valioso dentro de un proceso, por eso como parte de este 
estudio cuyo objetivo y misión es la de hacer un camino propicio para que los niños y los jóvenes de las comunidades rurales; además de tener una educación de calidad; es necesario adquirir como compromiso el buscar estrategias que conlleven al mejoramiento de una calidad humana, planteando conjuntamente con padres y alumnos dando alternativas en donde todos tengan la misma participación y por ende disfruten por igual los beneficios que este valor tiene los personajes.

\section{Referencias Bibliográficas}

Conde Gutiérrez Del Álamo, F., \& Santoro Domingo, P. (2012). Tipología, valores y preferencias de las personas con VIH e imaginarios de la infección: resultados de un estudio cualitativo. Revista española de salud pública, 86(2), 139-152.

Fouratt, C. (2011). Por el amor y la tierra: las inversiones emocionales de los migrantes nicaragüenses. Anuario de Estudios Centroamericanos, 38(1-2), 193-212.

Gimeno, J. (2008). El valor del tiempo en educación. Madrid: Morata.

Lourenço Suzana Cristina (2005). La educación ambiental bajo la visión de los sistemas no- formales e informales de formación educativa.

Martín, C. C. (2002). Educación ambiental y cambio de valores en la sociedad. Crónica bibliográfica. Observatorio medioambiental, 5, 357-364.

Martí J. (1963). 1973. Obras Completas. XXIX Tomos. Editorial Nacional de Cuba, La Habana. Cuba.

Rodríguez, J. M. M., \& Migueláñez, S. O. (2010). ADOLESCENCIA, TIEMPO LIBRE Y EDUCACIÓN. UN ESTUDIO CON ALUMNOS DE LA ESO (ADOLESCENCE, FREE TIME AND EDUCATION. A STUDY WITH STUDENTS FROM ESO COMPULSORY SECONDARY EDUCATION). Educación XX1, 13(2), 139..

Rodríguez, Z. A. I. D. A. (1985). Ciencia y valor. Filosofía y Ciencia. La Habana: Editorial de Ciencias Sociales. Segura, M., Mesa, J., \& ARCAS, M. (1997). Programa de competencia social, primero y segundo ciclo de primaria. Zubiri, X. (2002). Cinco lecciones de filosofía. Alianza Editorial. 